\title{
Transanal Assisted Resection with Closure of Anal Canal for Lower Rectal Diseases
}

\author{
HIDEJIRO KAWAHARA ${ }^{1}$, TADASHI AKIBA ${ }^{1}$ and KATSUHIKO YANAGA ${ }^{2}$ \\ ${ }^{1}$ Department of Surgery, Kashiwa Hospital, Jikei University School of Medicine, Chiba, Japan; \\ ${ }^{2}$ Department of Surgery, Jikei University School of Medicine, Tokyo, Japan
}

\begin{abstract}
Background/Aim: Abdominoperineal resection $(A P R)$ has been performed for lower rectal cancer, anal cancer and inflammatory diseases, but is associated with postoperative complications such as inflammation of pelvic dead space or perineal wound infection. We are performing a novel procedure (transanal assisted resection with closure of anal canal, TARC) that resects the intestine by using transanal assist and close the anal canal for patients who do not require resection of the anus and anal canal. Patients and Methods: From January 2015 to March 2017, nine patients (seven males, two females) underwent this procedure in our hospital for rectal cancer, recurrent rectal cancer, postoperative refractory pouchitis, and cervical cancer invaded to the rectum. The three patients diagnosed with rectal cancer underwent this procedure by a completely laparoscopic abdominal approach. Results: None developed postoperative dead space inflammation or perineal wound infections. Conclusion: TARC seems to allow improved postoperative quality of life as compared to APR.
\end{abstract}

Abdominoperineal resection (APR) introduced by Miles in 1908 (1) is a procedure to resect the anus with the anal canal and the surrounding tissue. APR has been performed for lower rectal cancer, anal cancer and inflammatory diseases (2) for more than one-hundred years, but is associated with postoperative complications such as inflammation of pelvic dead space or perineal wound infection (3). We are currently performing a novel procedure (transanal assisted resection with closure of anal canal, TARC) that resects the intestine by using transana

Correspondence to: Associate Prof. Hidejiro Kawahara, Department of Surgery, Kashiwa Hospital, Jikei University School of Medicine, 163-1 Kashiwashita, Kashiwashi, Chiba 277-8567, Japan. Tel: +81 471641111 Ext. 3421, Fax: +81 471633488, e-mail: kawahide@jikei.ac.jp

Key Words: Transanal resection, anus-preserving, rectal cancer, pouchitis. assist like the procedure so called intersphincteric resection (ISR) (4) (Figure 1) and close the anal canal for patients who do not require resection of the anus and anal canal (Figure 2).

\section{Patients and Methods}

From January 2015 to March 2017, nine patients underwent this procedure in our hospital for rectal cancer $(n=3)$, recurrent rectal cancer $(n=2)$, postoperative refractory pouchitis $(n=3)$, and cervical cancer invaded to the rectum $(n=1)$. The three patients diagnosed with rectal cancer underwent this procedure by a completely laparoscopic abdominal approach.

Surgical technique. At first, the rectum or neorectum is mobilized transabdominally to the upper border of the anal canal. After dilatation of the anus using the anal retractor, the anal canal is incised at the dentate line and mobilized with the internal sphincter cranially. After the resection of the rectum or neorectum with a part of the anal canal, the anal canal is obliterated by three interrupted 2-0 absorptive sutures. Finally, the anus is closed using 3-0 absorbable cross sutures at the dentate line (Figure 3 ). After tying the sutures, the anus looks untouched by surgery (Figure 4).

\section{Results}

The patients had a mean age of 55.4 (range=41-78) years, and 7 of them were male. The mean postoperative hospital stay was 11.6 (range $=10-16)$ days. None developed postoperative inflammation of pelvic dead space or perineal wound infections. The three patients underwent this procedure laparoscopically and their colostomy was created at the left lower quadrant to replace the inserted port position, had only four port incisions and a stoma in their abdomen after this procedure because a surgical specimen is removed through the anus. All nine patients could sit down easily like before surgery, starting the next day after surgery.

\section{Discussion}

One of the most important problems after APR is the tissue defect which could lead to postoperative complications. APR 


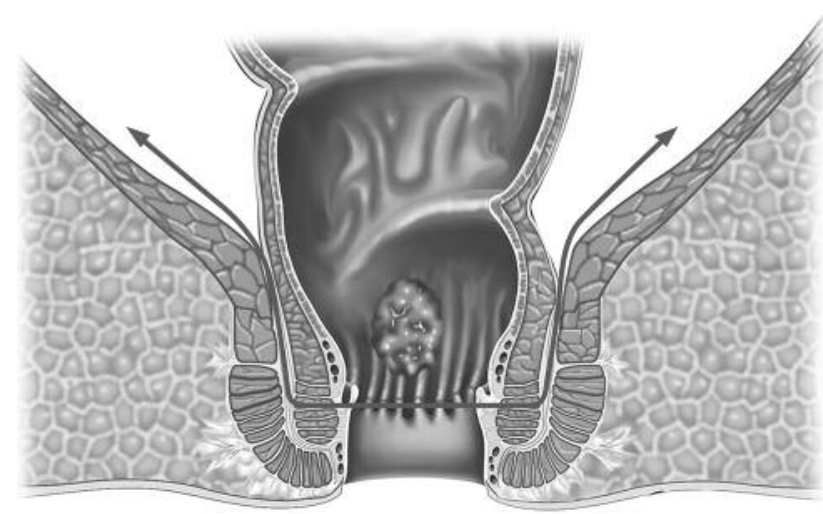

Figure 1. The intestine is resected by using transanal assist like the procedure so called intersphincteric resection.

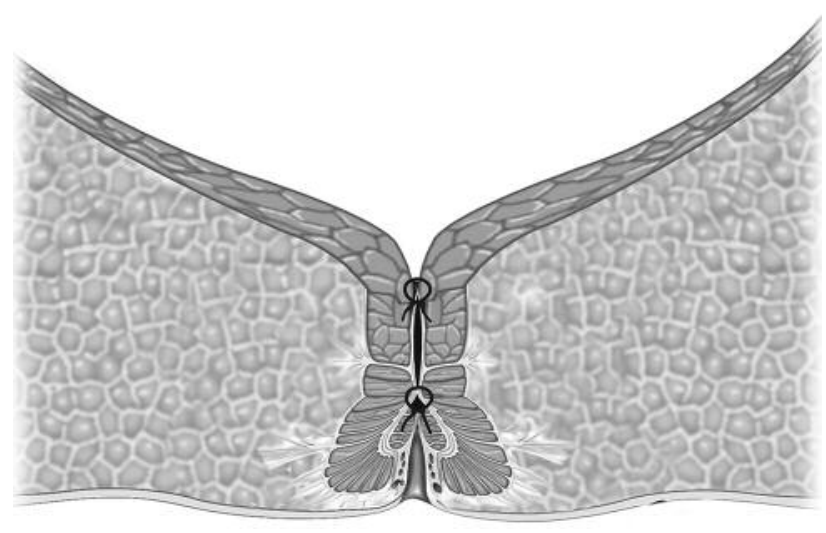

Figure 2. After the resection, the anal canal is closed by absorbable sutures.

involves resection of the rectum, anal canal, and perineal skin. When APR is chosen for cervical cancer with direct invasion of the rectum, tissue defect is too large, and requires plastic surgery for reconstruction, but postoperative wound infection is very common. We performed TARC for a patient with cervical cancer invading the rectum, who did not require plastic surgery for reconstruction because her tissue defect was not extensive.

Another problem after APR is perineal wound pain. Patients after APR cannot sit down after surgery without doughnut-form cushion because of perineal wound pain. All nine patients who underwent TARC could sit down easily like before surgery, starting the next day after surgery. The recovery after TARC seemed to be like in patients who underwent low anterior resection. This anus-preserving

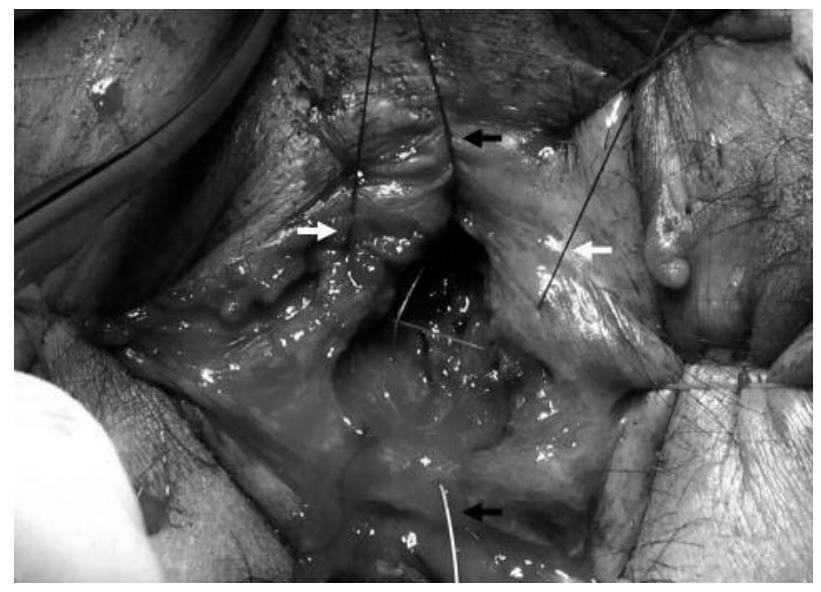

Figure 3. The anus is closed using 3-0 absorbable crossing sutures at the dentate line. The black arrows indicate the vertical suture, and the white arrows indicate the horizontal suture.

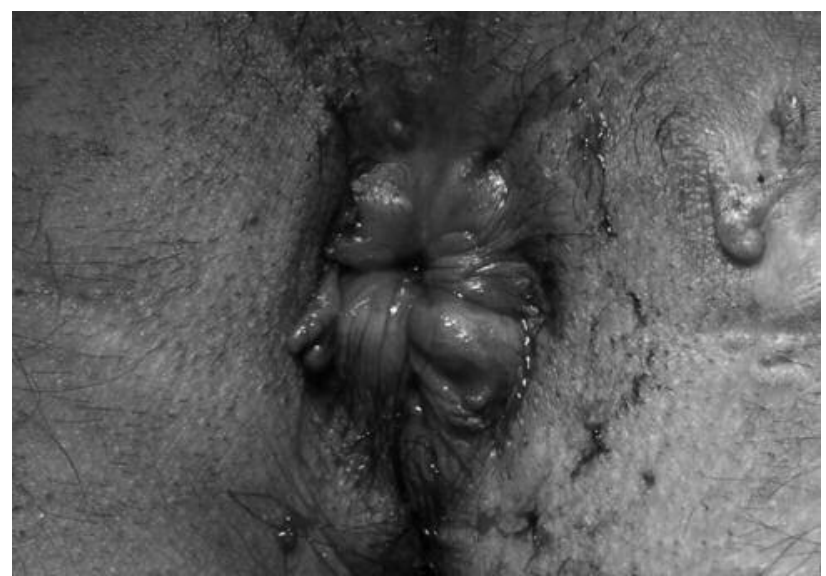

Figure 4. After closing with the cross sutures, the anus appears to be like before surgery.

operation has no defecation function however it has the possibility to reduce postoperative perineal wound pain and surgical site infection.

With regard to surgical techniques, there is a knack in the anal closure method. When a circular wound is sutured to the longitudinal direction or the transverse direction, the wound shows redundancy at the edge. Therefore, our crossing suture is preferable.

In conclusion, TARC that preserves the anus in selected patients, seems to allow improved postoperative quality of life compared to APR. 


\section{References}

1 Miles WE: A method of performing abdominal excision for carcinoma of the rectum and of the terminal portion of the pelvic colon. Lancet 2: 1812-1813, 1908.

2 Mauvais F, Sabbagh C, Brehant O, Viart L, Benhaim T, Fuks D, Sinna R and Regimbeau JM: The current abdominoperineal resection: oncological problems and surgical modifications for low rectal cancer. J Visc Surg 148: e85-93, 2011.
3 Althumairi AA, Canner JK, Gearhart SL, Safar B, Sacks J and Efron JE: Predictors of perineal wound complications and prolonged time to perineal wound healing after abdominoperineal resection. World J Surg 40: 1755-1762, 2016.

4 Lyttle JA and Parks AG: Intersphincteric excision of the rectum. Br J Surg 64: 413-416, 1977.

Received August 1, 2017

Revised August 21, 2017

Accepted August 22, 2017 